\title{
Singular orbits and Baker domains
}

\author{
Lasse Rempe ${ }^{1}$ (B)
}

Received: 15 September 2020 / Revised: 1 December 2020 / Accepted: 9 December 2020 /

Published online: 4 February 2021

(c) The Author(s) 2021

\begin{abstract}
We show that there is a transcendental meromorphic function with an invariant Baker domain $U$ such that every singular value of $f$ is a super-attracting periodic point. This answers a question of Bergweiler from 1993. We also show that $U$ can be chosen to contain arbitrarily large round annuli, centred at zero, of definite modulus. This answers a question of Mihaljević and the author from 2013, and complements recent work of Barański et al concerning this question.
\end{abstract}

Mathematics Subject Classification Primary 37F10; Secondary 30D05 - 37F31

\author{
I long for the Person from Porlock \\ To bring my thoughts to an end, \\ I am becoming impatient to see him \\ I think of him as a friend.
}

Stevie Smith

\section{Introduction}

Let $f: \hat{\mathbb{C}} \rightarrow \hat{\mathbb{C}}$ be a rational function of degree at least 2 . The Fatou set $F(f)$ consists of those points $z \in \mathbb{C}$ near which the iterates of $f$ form a normal family in the sense of Montel. In other words, these are the points at which the dynamics generated by $f$ is stable under small perturbations. A connected component of $F(f)$ is called a Fatou component; such a component is invariant if $f(U) \subset U$. An invariant Fatou component may be an immediate basin of attraction of an attracting or parabolic fixed point, or a simply or doubly connected domain on which $f$ is conjugate to an irrational rotation.

It was shown by Fatou $[8, \S 30-31]$ that there is a close relationship between invariant Fatou components and the critical values of $f$. Indeed, every attracting or parabolic

Communicated by Ngaiming Mok.

$\bowtie \quad$ Lasse Rempe

1.rempe@liverpool.ac.uk

1 Department of Mathematical Sciences, University of Liverpool, Liverpool L69 7ZL, UK 
basin must contain a critical value, and the boundary of any rotation domain is contained in the postcritical set $\mathcal{P}(f)$, i.e., the closure of the union of all critical orbits of $f$. See Lemmas 8.5, 15.7 and Theorems 10.15, 11.17 of [10].

These relationships carry over to the case of a transcendental meromorphic function $f: \mathbb{C} \rightarrow \hat{\mathbb{C}}$, with the set of critical values replaced by the set $\operatorname{sing}\left(f^{-1}\right)$ of critical or asymptotic values of $f$, and the postcritical set by the postsingular set defined analogously. In this setting, there is another possible type of invariant Fatou component: An invariant Baker domain, in which the iterates converge to the essential singularity at $\infty$. Bergweiler [3, Question 4] asked whether there is a relation between $\operatorname{sing}\left(f^{-1}\right)$ and the boundary of such a Baker domain. He also asked a more precise version of this question [3, Question 5]:

Question 1.1 Is it possible that a meromorphic function $f$ has Baker domains if the forward orbit of $z$ is bounded for all $z \in \operatorname{sing}\left(f^{-1}\right)$ ?

We give an affirmative answer.

Theorem 1.2 (Baker domains and super-attracting points) There is a transcendental meromorphic function $f: \mathbb{C} \rightarrow \hat{\mathbb{C}}$ with a Baker domain such that every point of $\operatorname{sing}\left(f^{-1}\right)$ is a super-attracting periodic point of period 2 .

Regarding [3, Question 4], Bergweiler [4, Theorem 3] obtained the following answer when $f$ is a transcendental entire function: If $U$ contains no critical or asymptotic values of $f$, then there exists a sequence $\left(p_{n}\right)_{n=0}^{\infty}$ of postsingular points $p_{n} \in \mathcal{P}(f)$ of $f$ such that

(a) $\left|p_{n}\right| \rightarrow \infty$;

(b) $\frac{\operatorname{dist}\left(p_{n}, U\right)}{\left|p_{n}\right|} \rightarrow 0$; and

(c) $\lim _{n \rightarrow \infty}\left|p_{n+1}\right| /\left|p_{n}\right|=1$.

On the other hand [4, Theorem 1], there is an entire function with a Baker domain $U$ such that $\operatorname{dist}(\mathcal{P}(f), \partial U)>0$, where dist denotes Euclidean distance.

For Baker domains of general transcendental meromorphic functions, it was shown in [11, Theorem 1.5] that an analogue of [4, Theorem 3] holds, without the hypothesis that $U$ contains no critical or asymptotic values, where condition (c) is replaced by the weaker

$$
\limsup _{n \rightarrow \infty} \frac{\left|p_{n+1}\right|}{\left|p_{n}\right|}<\infty .
$$

The article [11] also poses the following question.

Question 1.3 ( [11, Remark on p. 1603]) Can (1.1) be replaced by (c)?

In [7, Theorem $\mathrm{A}]$, it is shown that the answer is positive if $\mathbb{C} \backslash U$ has an unbounded connected component. Here we show that the answer to Question 1.3 is negative in general. 
Theorem 1.4 (Annuli of definite modulus) Let $\rho>0$. Then the function in Theorem 1.2 can be chosen such that there is a sequence $R_{j} \rightarrow \infty$ with

$$
\left\{z \in \mathbb{C}: R_{j}<|z|<\rho R_{j}\right\} \subset U \text { for all } j .
$$

The construction in our paper is inspired by those of Rippon and Stallard [12, Theorem 1.2] and Barański et al. [6, Theorem C]. Both of these construct meromorphic functions with multiply-connected Baker domains, obtained from an affine map by inserting poles at a sequence of points tending to infinity. We shall use quasiconformal surgery instead of explicit formulae, which allows us to obtain the required control of the singular orbits of $f$.

\section{Notation}

$\mathbb{C}, \hat{\mathbb{C}}$ and $\mathbb{D}$ denote the complex plane, Riemann sphere and unit disc, respectively. The Euclidean disc of radius $r$ around $z \in \mathbb{C}$ is denoted by $D(z, r)$.

\section{Quasiconformal surgery}

The function in Theorem 1.2 is constructed by a "cut and paste" quasiconformal surgery (see [5, Chapter 7]), starting from the linear map

$$
f_{0}(z):=\mu z
$$

where $\mu>1$. Consider the closed and connected set

$$
U_{0}:=\{z \in \mathbb{C}: \operatorname{Re} z \geq 0\} \cup \bigcup_{j \geq 0}\left\{z \in \mathbb{C}: \mu^{j} \leq|z| \leq \mu^{j+\frac{1}{2}}\right\},
$$

which is forward-invariant under $f_{0}$. Also let $D_{0}$ be a closed disc

$$
D_{0}=\overline{D\left(\zeta_{0}, r_{0}\right)} \subset\{z \in \mathbb{C}: \operatorname{Re} z<0 \text { and } \sqrt{\mu}<|z|<\mu\} \subset \mathbb{C} \backslash U_{0}
$$

If we define

$$
D_{j}:=f_{0}^{j}\left(D_{0}\right)=\overline{D\left(\mu^{j} \cdot \zeta_{0}, \mu^{j} \cdot r_{0}\right)}=: \overline{D\left(\zeta_{j}, r_{j}\right)}
$$

then all $D_{j}$ are disjoint from $U_{0}$, and from each other.

We construct our functions from $f_{0}$ by a quasiconformal surgery that inserts poles in the discs $D_{j}$. The following makes this procedure precise.

Proposition 2.1 (Quasiconformal surgery of $f_{0}$ ) Let $\mu>1$, and let $f_{0}, U_{0}$ and $\left(D_{j}\right)_{j=0}^{\infty}$ be defined as above. Let $K>1$, and let

$$
h_{j}: D_{j} \rightarrow \hat{\mathbb{C}}
$$


be a sequence of $K$-quasiregular functions such that each $h_{j}$ extends continuously to д $D_{j}$ with $h_{j}=f_{0}$ on $\partial D_{j}$. Then

$$
F: \mathbb{C} \rightarrow \hat{\mathbb{C}} ; \quad z \mapsto \begin{cases}h_{j}(z) & \text { if } z \in D_{j} \\ f_{0}(z) & \text { if } z \notin \bigcup_{j} D_{j} .\end{cases}
$$

is $K$-quasiregular, with no finite asymptotic values, and every critical value of $F$ is a critical value of some $h_{j}$.

Suppose furthermore that, for infinitely many $j$, the map $h_{j}$ is not a homeomorphism $h_{j}: D_{j} \rightarrow D_{j+1}$, and that for every $j$ there is an open set $W_{j}$ with the following properties.

(a) The dilatation of $h_{j}$ is supported on $W_{j}$.

(b) $F$ is conformal on $F^{n}\left(W_{j}\right)$ for all $n \geq 1$.

Then there is a quasiconformal homeomorphism $\psi: \mathbb{C} \rightarrow \mathbb{C}$ with $\psi(0)=0$ such that

$$
f:=\psi \circ F \circ \psi^{-1}
$$

is a transcendental meromorphic function. Moreover, $\psi$ is conformal on $U_{0}$, and $\psi\left(U_{0}\right)$ is contained in an invariant Baker domain $U$ of $f$.

Proof That $F$ is $K$-quasiregular follows from Royden's glueing lemma [2, Lemma 2], or the quasiconformal removability of quasiarcs [5, Theorem 1.19]. Any curve to infinity must intersect $U_{0}$, and therefore $F$ is unbounded on any such curve. In particular, $F$ has no finite asymptotic values. As $F=f_{0}$ on $U_{0}$, every critical value of $F$ must come from one of the $h_{j}$.

Now suppose that the additional conditions hold. Since $h_{j}$ maps $\partial D_{j}$ to $\partial D_{j+1}$ in one-to-one fashion, if $h_{j}$ is not a homeomorphism, then $h_{j}$ must have at least one pole in $D_{j}$. As this happens for infinitely many $j$, we see that $F$ has infinitely many poles. Let $\omega_{0}:=F_{0}^{*}(0)$ be the complex dilatation of $F$. By (b), the restriction of $\omega_{0}$ to

$$
W:=\bigcup_{j, n \geq 0} F^{n}\left(W_{j}\right)
$$

is forward-invariant under $F$. As $F$ is meromorphic outside of $W$, we may extend $\left.\omega_{0}\right|_{W}$ by pull-back to the grand orbit of $W$. Extending the resulting differential by the standard complex structure, we obtain an $F$-invariant measurable Beltrami differential $\omega$ on all of $\hat{\mathbb{C}}$. Observe that $\omega \equiv 0$ on $U_{0}$.

By the measurable Riemann mapping theorem, there is a quasiconformal homeomorphism $\psi: \mathbb{C} \rightarrow \hat{\mathbb{C}}$ that solves the Beltrami equation for $\omega$. Then $\psi$ is conformal on $U_{0}$. Moreover, the map $f$ defined by (2.3) is meromorphic; it is transcendental since $F$ has infinitely many poles. Let $z \in \psi\left(U_{0}\right)$; say $z=\psi(\zeta)$ with $\zeta \in U_{0}$. Then

$$
\begin{aligned}
& f_{0}(z)=\psi\left(F\left(\psi^{-1}(z)\right)\right)=\psi(F(\zeta)) \in \psi\left(U_{0}\right), \text { and } \\
& f_{0}^{n}(z)=\psi\left(F^{n}\left(\psi^{-1}(z)\right)\right)=\psi\left(F^{n}(\zeta)\right)=\psi\left(f_{0}^{n}(\zeta)\right) \rightarrow \infty
\end{aligned}
$$

So $\psi\left(U_{0}\right)$ is contained in a Baker domain of $f$, as claimed. 
Proposition 2.2 (Large annuli in $U$ ) For every $\rho>1$, there is $\mu>1$ with the following property. Let $f_{0}(z)=\mu \cdot z$, and let $f$ be obtained from $f_{0}$ as in Proposition 2.1. Then $f$ satisfies (1.2) for a sequence $R_{j} \rightarrow \infty$.

Proof Recall that $U_{0}$ contains arbitrarily large round annuli, each of modulus at least $M:=(\log \mu) / 2$. If $M \geq \Lambda(\rho)$, where $\Lambda(\rho)$ is the modulus of the Teichmüller annulus $[1, \S 4-11]$, then any annulus that separates 0 from $\infty$ and has modulus at least $M$ contains a round annulus of modulus $\log \rho$ centred at zero. Hence we can take $\mu=e^{2 \Lambda(\rho)}$.

\section{Proof of Theorem 1.2}

To prove Theorem 1.2, we construct a suitable sequence of functions $h_{j}$ to use in Proposition 2.1. The idea is that each $h_{j}$ has critical values in $D_{j+1}$, which are then mapped back to the original critical points by $h_{j+1}$. We begin with the following, where $f_{0}$ is again given by (2.1).

Proposition 3.1 Let $\Delta=D(\zeta, r) \subset \mathbb{C}$ be a round disc, and let $K>1,0<\eta<r$, and $0<\vartheta<\mu \cdot \eta$. For every $a \in \mathbb{C} \backslash f_{0}(\Delta)$, there is a $K$-quasiregular map

$$
g: \Delta \rightarrow \hat{\mathbb{C}}
$$

such that

(1) $g$ extends continuously to $\partial \Delta$, where it agrees with $f_{0}$.

(2) $g(\zeta)=a$.

(3) $g$ has exactly two critical points, $c_{1}$ and $c_{2}$, with $g\left(c_{1}\right) \neq g\left(c_{2}\right)$.

(4) Each critical point $c_{j}$ satisfies $g\left(c_{j}\right) \in D\left(f_{0}(\zeta), \vartheta\right)$.

(5) $g$ is meromorphic on $D(\zeta, \eta)$.

(6) $g$ is injective (and hence quasiconformal) on $\Delta \backslash D(\zeta, \eta)$. Furthermore, g satisfies $\mu r>\left|g(z)-f_{0}(\zeta)\right|>\vartheta$ for $r>|z-\zeta| \geq \eta$.

Proof Set

$$
\alpha:=\frac{a}{r \mu}-\frac{\zeta}{r} \neq 0
$$

Let $\varepsilon>0$, and consider the map

$$
\varphi=\varphi_{\alpha, \varepsilon}: \mathbb{D} \rightarrow \hat{\mathbb{C}} ; \quad z \mapsto z+\frac{\varepsilon \alpha}{\alpha z+\varepsilon}=z+\frac{\varepsilon}{z-p},
$$

where $p=-\varepsilon / \alpha$. Then $\varphi(0)=\alpha$ and $\varphi(p)=\infty$. By direct calculation, $\varphi$ has two simple critical points at $p \pm \sqrt{\varepsilon}$, with critical values at $p \pm 2 \sqrt{\varepsilon}$.

Now define $A: \hat{\mathbb{C}} \rightarrow \hat{\mathbb{C}}$ to be the affine map taking $\mathbb{D}$ to $\Delta$; i.e., $A(z)=r \cdot z+\zeta$. Define

$$
g_{\varepsilon}: D(\zeta, \eta) \rightarrow \hat{\mathbb{C}} ; \quad z \mapsto \mu \cdot A\left(\varphi_{\alpha, \varepsilon}\left(A^{-1}(z)\right)\right)
$$


For all sufficiently small $\varepsilon, g_{\varepsilon}$ satisfies (3),(4) and (5) . Furthermore,

$$
g_{\varepsilon}(\zeta)=\mu \cdot(r \alpha+\zeta)=a,
$$

so $g$ also satisfies (2).

Set $\Delta_{\eta}:=D(\zeta, \eta)$. As $\varepsilon \rightarrow 0, g_{\varepsilon} \rightarrow f_{0}$ uniformly on a neighbourhood of $\partial \Delta_{\eta}$. In particular, for sufficiently small $\varepsilon, g_{\varepsilon}$ is conformal on a neighbourhood of $\partial \Delta_{\eta}$, and satisfies

$$
\mu r>\left|g_{\varepsilon}(z)-f_{0}(\zeta)\right|>\vartheta
$$

when $z \in \partial \Delta_{\eta}$. It follows that there is a quasiconformal homeomorphism $G$ that maps the round annulus $\Delta \backslash \overline{\Delta_{\eta}}$ to the annulus bounded by $f_{0}(\partial \Delta)$ and $g_{\varepsilon}\left(\partial \Delta_{\eta}\right)$, and which agrees with $f_{0}$ on $\partial \Delta$ and with $g_{\varepsilon}$ on $\partial \Delta_{\eta}$; see [9]. We define $g$ to agree with $g_{\varepsilon}$ on $D_{\eta}$ and with $G$ outside; then $g$ satisfies (1)-(6).

Finally, we claim that the dilatation of $G$, and thus of $g$, tends to 1 as $\varepsilon \rightarrow 0$. Indeed, this follows readily from the construction in [9]. Alternatively, for small $\varepsilon$, we can define $G$ directly by linear interpolation on each radius of $\Delta \backslash \overline{\Delta_{\eta}}$. An elementary calculation shows that the dilatation of $G$ tends to 1 . Hence, for sufficiently small $\varepsilon$, $g$ is $K$-quasiconformal as claimed.

For the remainder of the paper, let $D_{j}$ denote the discs introduced in Sect. 2.

Proposition 3.2 For any $K>1$, there is a sequence of $K$-quasiregular maps

$$
h_{j}: D_{j} \rightarrow \hat{\mathbb{C}}
$$

with the following properties for all $j$.

(a) For every $j$, the map $h_{j}$ has at least one critical point.

(b) If $c$ is a critical point of $h_{j}$, then $h_{j}(c) \in D_{j+1}$ and $h_{j+1}\left(h_{j}(c)\right)=c$.

Furthermore, for each $j$ there are open sets $V_{j} \subset W_{j} \subset D_{j}$ such that

(c) $D_{j} \backslash V_{j}$ is compact;

(d) $h_{j}$ is injective on $W_{j}$;

(e) $h_{j}(z)=f_{0}(z)$ for $z \in V_{j}$;

(f) $h_{j}\left(W_{j}\right) \subset V_{j+1}$;

(g) The dilatation of $h_{j}$ is supported on $W_{j}$.

Proof The sequence of functions is constructed inductively, in such a way as to ensure the following inductive hypotheses. For $j \geq 0$, let $\mathcal{C}_{j} \subset D_{j}$ be the set of critical points of $h_{j}$ and $\Omega_{j+1}=h_{j}\left(\mathcal{C}_{j}\right) \subset \hat{\mathbb{C}}$ the set of critical values. The construction will ensure the following inductive hypotheses for $j \geq 0$ :

(A) $\Omega_{j+1} \subset D_{j+1}$.

(B) $h_{j}: \mathcal{C}_{j} \rightarrow \Omega_{j+1}$ is bijective.

(C) $h_{j}\left(W_{j}\right) \subset D_{j+1}$ and $\overline{h_{j}\left(W_{j}\right)} \cap \Omega_{j+1}=\emptyset$. 
To anchor the recursive construction, we also set $\Omega_{0}:=\left\{\zeta_{0}\right\}$. This means that (A) holds also for $j=-1$.

Now suppose that $j \geq 0$ is such that $h_{i}$ has been constructed for $i<j$, satisfying the inductive hypotheses. To define $h_{j}$, let $\omega_{j}^{1}, \ldots, \omega_{j}^{m}$ be the elements of $\Omega_{j}$. Choose pairwise disjoint small discs $\Delta_{j}^{i}=D\left(\omega_{j}^{i}, \rho_{j}^{i}\right) \Subset D_{j}$, for $j=1, \ldots, m$; this is possible by (A). If $j>0$, then by (C) we may also ensure that the closures of the $\Delta_{j}^{i}$ do not intersect $\overline{h_{j-1}\left(W_{j-1}\right)}$. Set

$$
V_{j}:=D_{j} \backslash \bigcup_{i=1}^{m} \overline{\Delta_{j}^{i}} \supset h_{j-1}\left(W_{j-1}\right) .
$$

For each $i=1, \ldots, m$, apply Proposition 3.1 to $\Delta=\Delta_{j}^{i}$, where $a=c_{j-1}^{i} \in \mathcal{C}_{j-1}$ is such that

$$
h_{j-1}\left(c_{j-1}^{i}\right)=\omega_{j}^{i}
$$

if $j>0$, and $c_{-1}^{1}=0$ otherwise. We may choose $\vartheta$ and $\eta=\eta_{j}^{i}$ arbitrary, subject to the conditions given in the proposition. Let $g_{j}^{i}$ denote the maps obtained. We define

$$
\begin{gathered}
h_{j}(z):=\left\{\begin{array}{ll}
f_{0}(z) & \text { if } z \in V_{j} ; \\
g_{j}^{i}(z) & \text { if } z \in \overline{\Delta_{j}^{i}}
\end{array} \quad\right. \text { and } \\
W_{j}:=D_{j} \backslash \bigcup_{i=1}^{m} \overline{D\left(\omega_{j}^{i}, \eta_{j}^{i}\right)} \supset V_{j} .
\end{gathered}
$$

We claim that $h_{j}$ satisfies (A)-(C). For the remainder of the proof, references to (1)(6) shall mean the corresponding properties of the $g_{j}^{i}$ established in Proposition 3.1.

The critical points and critical values of $h_{j}$ are exactly those of the maps $g_{j}^{i}$. By (4), the critical values of $g_{j}^{i}$ are in $f_{0}\left(\Delta_{j}^{i}\right) \subset f_{0}\left(D_{j}\right)=D_{j+1}$, establishing (A). Furthermore, by (3), $g_{j}^{i}$ has exactly two different critical points, with different critical values belonging to $f_{0}\left(\Delta_{j}^{i}\right)$. Since different $\Delta_{j}^{i}$ are pairwise disjoint, and $f_{0}$ is injective, claim (B) follows. Finally, (C) is an immediate consequence of (4) and (6) .

This completes the construction of the $h_{j}$. By (1) and Royden's glueing lemma (or the quasiconformal removability of quasiarcs), the map $h_{j}$ is indeed a $K$-quasiregular map. It remains to establish (a)-(g).

(a) Each $h_{j}^{i}$ has exactly two critical values, and $\# \Omega_{0}=1$. It follows inductively that $\# \Omega_{j}=2^{j}$ for all $j$, and in particular each $h_{j}$ has at least one critical point.

(b) If $c \in \mathcal{C}_{j}$ is a critical point of $h_{j}$, then we must have $c=c_{j}^{i}$ for some $i$, by (3.2) and (B). So by (2),

$$
h_{j+1}\left(h_{j}(c)\right)=h_{j+1}\left(h_{j}\left(c_{j}^{i}\right)\right)=g_{j+1}^{i}\left(\omega_{j+1}^{i}\right)=c_{j}^{i}=c .
$$

(c) By definition of $V_{j}$ in (3.1), $D_{j} \backslash V_{j}$ is compact. 
(d) $\left.h_{j}\right|_{W_{j}}$ is injective by (3.4) and (6) .

(e) By (3.3), $h_{j}=f_{0}$ on $V_{j}$.

(f) By (3.1), $V_{j+1} \supset h_{j}\left(W_{j}\right)$.

(g) By (5), the dilatation of $h_{j}$ is supported on $W_{j}$.

Proof of Theorems 1.2 and 1.4 Choose $\mu$ as in Proposition 2.2, and let $\left(h_{j}\right)_{j=0}^{\infty}$ be as in Proposition 3.2. Let $F$ be the quasiregular map defined by (2.2). Then $F^{2}(c)=c$ for every critical point $c$ of $F$.

The dilatation of $h_{j}$ is supported on $W_{j}$, and $h_{j}$ is conformal on $\overline{V_{j}}$. Moreover, $F^{n}\left(W_{j}\right) \subset V_{n+j}$ for all for all $\mathrm{n} \geq 1$ and $\mathrm{j} \geq 0$, so $W j$ satisfies the conditions of Proposition 2.1. Let $f$ be the meromorphic function (2.3); then $f$ has a Baker domain $U$ and satisfies $f^{2}(c)=c$ for every critical point of $f$. This completes the Proof of Theorem 1.2.

Furthermore, by Proposition 2.2, the function also satisfies (1.2) for a sequence $R_{j} \rightarrow \infty$, proving Theorem 1.4.

Acknowledgements I am grateful to Bogusława Karpińska, whose excellent presentation about [7] at the 2020 online conference On geometric complexity of Julia sets - II inspired this work. I also thank her and Walter Bergweiler for interesting discussions following the talk, and impan and the Banach Centre in Warsaw for hosting the above conference. Finally, I am grateful to the referee for their thoughtful comments, which improved the paper.

Open Access This article is licensed under a Creative Commons Attribution 4.0 International License, which permits use, sharing, adaptation, distribution and reproduction in any medium or format, as long as you give appropriate credit to the original author(s) and the source, provide a link to the Creative Commons licence, and indicate if changes were made. The images or other third party material in this article are included in the article's Creative Commons licence, unless indicated otherwise in a credit line to the material. If material is not included in the article's Creative Commons licence and your intended use is not permitted by statutory regulation or exceeds the permitted use, you will need to obtain permission directly from the copyright holder. To view a copy of this licence, visit http://creativecommons.org/licenses/by/4.0/.

\section{References}

1. Ahlfors, L.V.: Conformal invariants: topics in geometric function theory, McGraw-Hill Book Co., New York-Düsseldorf-Johannesburg, 1973, McGraw-Hill Series in Higher Mathematics

2. Bers, L.: On moduli of Kleinian groups. Russian Math. Surv. 29(2), 88-102 (1974)

3. Bergweiler, W.: Iteration of meromorphic functions. Bull. Am. Math. Soc. (N.S.) 29(2), 151-188 (1993)

4. Bergweiler, W.: Invariant domains and singularities. Math. Proc. Cambridge Philos. Soc 117(3), 525532 (1995)

5. Branner, B., Fagella, N.: Quasiconformal surgery in holomorphic dynamics, Cambridge Studies in Advanced Mathematics, vol. 141, Cambridge University Press, Cambridge (2014), With contributions by Xavier Buff, Shaun Bullett, Adam L. Epstein, Peter Haïssinsky, Christian Henriksen, Carsten L. Petersen, Kevin M. Pilgrim, Tan Lei and Michael Yampolsky

6. Barański, K., Fagella, N., Jarque, X., Karpińska, B.: Absorbing sets and Baker domains for holomorphic maps. J. Lond. Math. Soc(2) 92(1), 144-162 (2015)

7. Barański, K., Fagella, N., Jarque, X., Karpińska, B.: Fatou components and singularities of meromorphic functions. Proc. Roy. Soc. Edinburgh Sect. A 150(2), 633-654 (2020)

8. Fatou, P.: Sur les équations fonctionnelles (deuxième mémoire). Bulletin de la Société Mathématique de France 48, 33-94 (1920)

9. Lehto, O.: An extension theorem for quasiconformal mappings. Proc. London Math. Soc. 14a(3), 187-190 (1965) 
10. Milnor, J.: Dynamics in One Complex Variable. Annals of Mathematics Studies, vol. 160, 3rd edn. Princeton University Press, Princeton, NJ (2006)

11. Mihaljević-Brandt, H., Rempe-Gillen, L.: Absence of wandering domains for some real entire functions with bounded singular sets. Math. Ann. 357(4), 1577-1604 (2013)

12. Rippon, P.J., Stallard, G.M.: Singularities of meromorphic functions with Baker domains. Math. Proc. Cambridge Philos. Soc. 141(2), 371-382 (2006)

Publisher's Note Springer Nature remains neutral with regard to jurisdictional claims in published maps and institutional affiliations. 